\title{
Effectiveness of an App-based Intervention to Reduce Substance Use, Gambling and Digital Media Use in Vocational School Students: Study Protocol for a Randomized Controlled Trial
}

\author{
Nicolas Arnaud ( $\nabla$ n.arnaud@uke.de) \\ Universitatsklinikum Hamburg-Eppendorf https://orcid.org/0000-0002-3367-0815 \\ Johanna Weymann \\ Universitätsklinikum Hamburg-Eppendorf: Universitatsklinikum Hamburg-Eppendorf \\ Kirsten Lochbühler \\ Institut für Therapieforschung: Institut fur Therapieforschung \\ Benjamin Pietsch
}

Institute for Therapy Research: Institut fur Therapieforschung

\section{Monika Rossa}

Institut fur Therapieforschung

\section{Ludwig Kraus}

Institut fur Therapieforschung

\section{Rainer Thomasius}

Universitätsklinikum Hamburg-Eppendorf: Universitatsklinikum Hamburg-Eppendorf

\section{Reiner Hanewinkel}

Institute for Therapy Research: Institut fur Therapieforschung

\section{Matthis Morgenstern}

Institute for Therapy Research: Institut fur Therapieforschung

\section{Research Article}

Keywords: Prevention, vocational students, voluntary commitment, abstinence, substance use, internetrelated problems

Posted Date: February 21st, 2022

DOI: https://doi.org/10.21203/rs.3.rs-1229246/v1

License: (a) This work is licensed under a Creative Commons Attribution 4.0 International License. Read Full License 
Version of Record: A version of this preprint was published at Trials on April 8th, 2022. See the published version at https://doi.org/10.1186/s13063-022-06231-x. 


\section{Abstract}

\section{Background:}

Substance-related and addictive disorders are among the most common mental disorders in adolescence and young adulthood. Vocational school students are a risk group for problematic substance use and addictive behavior. However, the availability of evidence-based prevention concepts and programs is underdeveloped in the vocational school setting.

\section{Methods/design:}

A two-arm cluster randomized waitlist-controlled trial will be conducted to evaluate the effectiveness of an app-based intervention to decrease substance use, gambling and digital media use in vocational school students in Germany. Vocational students will participate in an app-based intervention that is designed to support voluntary commitment to abstain from or reduce substance or digital media use over a period of two weeks. The "education-as-usual" control arm will have access to the intervention after data collection is completed. Primary outcome measures will be use of alcohol, nicotine, and digital media 30 days after intervention. Several secondary outcome measures will also be included, such as cannabis consumption, gambling, symptoms of stress, physical activity, mindfulness, well-being, impulsivity and sensation seeking, and readiness to change. A total of 4,500 vocational students from 225 classes will be recruited and randomized across three German federal states.

\section{Discussion:}

This study protocol describes the design of an RCT testing the effectiveness of an app-based intervention to reduce addictive behaviors in vocational school students. It is expected that this approach will be feasible for and effective in the vocational school setting and that the study provides comprehensive information on the key factors involved in temporary abstaining or reducing substance or digital media use.

\section{Trial registration:}

German Register of Clinical Studies: No. DRKS00023788, registered on January 20, 2021

\section{Introduction}

\section{Background and rationale $\{6 a\}$}

Substance-related and addictive disorders typically have their onset in early adolescence and belong to the most common psychological disorders among emerging adults [1, 2]. In Germany, the group of 18 to 20 -year-olds has the highest risk of addiction diagnoses regarding alcohol (6.4\%), cannabis (1.5\%), amphetamines $(0.4 \%)$ and cocaine $(0.3 \%)$ according to population-based survey data [3]. Research has consistently shown that the period of emerging adulthood [4] is not only associated with a readiness to 
engage in risky and unhealthy behavior such as substance abuse but also with limited capacities for selfregulation and (substance use-related) habit formation [5].

The proportion of young people affected by addictive behaviors increases if non-substance-related or "behavioral" addictions, particularly internet-related disorders are also taken into account [6]. Gambling Disorder and (screen-related) Gaming Disorder seem to be similar to substance-related addictive disorders regarding their clinical appearance, etiology, comorbidity and therapeutic responsiveness $[7,8]$. Moreover, they are of increasing epidemiological relevance (even more so during the current COVID-19 pandemic conditions, see [9]) and have therefore recently been included in the revised classification of Substance-Related and Addictive Disorders in the 11th edition of the International Classification of Diseases (ICD-11) of the World Health Organization (WHO) (see [10]).

The school setting is particularly relevant for prevention efforts as it represents a central developmental context where a large number of individuals can be reached with comparatively little effort [11]. The majority of evaluated school-based preventive intervention-programs apply only to regular schools, while there has been a lack of comparable programs for vocational schools [12]. However, in Germany a substantial proportion of young people leaves the regular school system during adolescence and starts a vocational education, typically from the age of $16[13,14]$. Vocational education in Germany largely takes place in the "dual system", in which trainees are employed in a company and complete the practical part of their training there, while also attending vocational school, where the theoretical part of the training takes place.

Recent studies suggest that vocational students represent an important target group for prevention efforts and health promotion. In a survey including 5,688 German vocational students $40.5 \%$ of the participants reported a positive screening result for problematic alcohol use and $3.6 \%$ reported a level of cannabis use that puts them at serious risk for addiction [15]. The percentage of vocational students that reported daily smoking (41\%) was 2.4 times greater than their age-matched peers in the general population. With regard to addictive behaviors such as online and offline gambling and digital media use there are currently no data available that estimate the specific risks of vocational students. However, surveys indicate that media-related problems are particularly widespread among adolescents and young adults of typical vocational school age $[16,17]$.

A promising school-based prevention approach is a voluntary commitment to abstain from or reduce habitual behaviors like substance use. For example, one of the most widespread programs for the prevention of smoking in secondary schools in Germany is the smoke-free class competition "Be Smart Don't Start". At the core of the program is a joint voluntary commitment of the school class to not smoke for a period of six months. It focuses on influencing social norms, promoting self-regulation and addressing social influences by deploying cognitive-behavioral intervention techniques [18]. Overall, the evidence based on process and rigorous outcome evaluations, long-term and iatrogenic effects and costbenefit efficiency are convincing, even in comparison to other programs (see [19]). An adaptation of this approach has also been shown to be effective for the prevention of binge drinking among older 
adolescents in the regular school setting in a randomized study [20]. Further, the results of a recent controlled study in Germany [21] suggested that a 20-minute reduction of daily social media use over a period of two weeks is positively associated with well-being and a healthier lifestyle among students. Specifically, the reduction in social media use not only reduced social media use intensity and the level of addictive symptoms but also the amount of daily smoked cigarettes over a period of three months.

\section{Objectives $\{7\}$}

The present study aims to transfer the approach of voluntary abstinence to the vocational school setting and to evaluate the feasibility and effectiveness of this approach using a randomized design. It is expected that the intervention will increase awareness of habitual behaviors (e.g., substance and digital media use) and lead to a measurable reduction of these behaviors even after the end of the abstinence or reduction period.

\section{Trial design $\{8\}$}

The current study uses a cluster-randomized controlled trial with two arms, an intervention group (IG) and a waitlist control group (CG) with pre-post assessments. All study participants will be randomly assigned to one of the two groups on class level (Fig. 1).

\section{Methods: Participants, Interventions And Outcomes}

\section{Study setting $\{9\}$}

The study will be conducted in vocational schools in the three German federal states of SchleswigHolstein, Bavaria and Hamburg.

\section{Eligibility criteria $\{10\}$}

All vocational students will be eligible to participate in the study if they or one of their legal guardians (if the student is under the age of 16) provide written informed consent.

\section{Who will take informed consent? $\{26 \mathrm{a}\}$}

Trial participants and if under the age of 16 , their legal guardians, will be provided with sufficient verbal and written information about the study's purpose and procedures, information on confidentiality and data protection procedures, possible advantages and disadvantages of participation, and the option to withdraw from the study at any time and without any given reason. They are informed that participation in the study is completely voluntary. Written informed consent will be obtained from all participants prior to study enrollment.

\section{Additional consent provisions for collection and use of participant data and biological specimens $\{26 \mathrm{~b}\}$}

The consent includes participant's agreement on the collected data being used and published in an anonymized form for research purposes. This trial does not involve collecting biological specimens for 
storage.

\section{Interventions}

\section{Explanation for the choice of comparators $\{6 \mathrm{~b}\}$}

The comparator is an education as usual waitlist control group. All participants will be randomly assigned to either the app-based intervention group or the control group. The participants of the control group will continue routine activities during the (approximately) 6-week waiting period. After postassessment, participants of the control group will receive full access to the intervention app.

\section{Intervention description $\{11 \mathrm{a}\}$}

"Meine Zeit ohne" [my time without] (MZo) is an app-based intervention that aims to encourage users to voluntary abstain from an individually relevant habitual behavior or to reduce it to a degree that is subjectively considered significant or a "challenge" for a period of two weeks. After downloading the app (downloadable for devices running on iOS 11.0 or higher / Android 6 or higher) and log-in with a password, students can set their individual challenge. For the next two weeks participants receive push notifications on a daily basis and are asked for feedback about whether they have reached their goal of the preceding day. MZo primarily targets consumption behavior (both substance related and nonsubstance related behavior, i.e., digital/ screen based media related behavior such as gaming and/ or social media use).

\section{Criteria for discontinuing or modifying allocated interventions $\{11 \mathrm{~b}\}$}

Participants in the intervention group can discontinue the use of the intervention app at any time by deleting the app from their mobile device. The intervention, i.e. the use of the app does not require any contact to the study team.

\section{Strategies to improve adherence to interventions $\{11 \mathrm{c}\}$}

The implementation of the MZo-Challenge is entirely app-based. The effort required to use the app can be considered minimal. Adherence to the intervention is facilitated by an easy access procedure (log-in and user authentication is only required once after the download of the app) and daily push notifications. The app provides the opportunity to choose individual behavior change goals from a broad spectrum including an undefined goal category which can be specified by the individual user.

\section{Relevant concomitant care permitted or prohibited during the trial $\{11 \mathrm{~d}\}$}

Participation in the current trial has no impact on possible concomitant care during the time of the trial.

\section{Provisions for post-trial care $\{30\}$}


All participating schools are provided with contact information of the study sites and can access evidence-based information material on substance use and substance use prevention via the project website.

\section{Outcomes $\{12\}$}

\section{Primary outcomes}

Outcome measures at each time point are shown in Figure 2. Primary outcomes are self-reported use of alcohol, nicotine (cigarettes and/ or electronic cigarettes), digital media (gaming and social media) in the past month. Measures for alcohol use are based on [22] and include frequency of drinking ( $1=$ "never" to $5=$ "four times a week or more"), quantity of alcoholic drinks on a typical drinking day ( $1=$ " 1 or 2 alcoholic drinks" to $5=$ " 10 or more alcoholic drinks") and frequency of binge drinking (more than 6 alcoholic drinks on one occasion; $0=$ "never" to $4=$ "daily or almost daily"). Nicotine use is assessed based on students' reports on the number of days using cigarettes and/ or electronic cigarettes and their quantity per day. Problematic Internet gaming is assessed using the Internet Gaming Disorder ScaleShort-Form (IGDS-SF; [23]), German version ([24]; 9-items: e.g., "Have you continued your gaming activity despite knowing it was causing problems between you and other people?"; 1 = "never" to 5 = "very often"). Problematic social media use was assessed using an adapted version of the brief version of the Bergen Facebook Addiction Scale (BFAS; [25]; German version: [26]). The scale consists of 6-items (e.g., "Felt an urge to use social media more and more"; 1 = "very rarely" to 5 = "very often").

\section{Secondary outcomes}

A number of individual-level secondary outcome measures were selected based on their health-related relevance and associability with the conceptual intervention approach. These outcomes are:

- Problematic Cannabis Use (SDS, Severity of Dependence Scale, 5 items [27]; German version: [28]).

- Problematic Gambling (BBGS, Brief Biosocial Gambling Screen, 3 items [29])

- Psychological Stress (DASS-21, Depression Anxiety Stress Scales 21, subscale stress, 7 items [30]; German version: [31])

- Impulsivity and Sensation Seeking (SURPS, Substance Use Risk Profile Scale, subscales impulsivity and sensation seeking, 2 items each [32])

- Mindfulness Skills (CAMM, Child and Adolescent Mindfulness Measure, 10 items [33])

- Physical activity (past month frequency of physical activity/ sports, single item, self-constructed)

- Positive mental Health (PMH, Positive Mental Health-scale, 9 items [34])

- Life satisfaction (L1, General Life Satisfaction Short Scale, single item [35], German version [36])

- General Self-efficacy (ASKU, Short Scale for Measuring General Self-efficacy Beliefs, 3 items [37])

- Readiness and confidence to quit or reduce use of alcohol, nicotine, cannabis, gaming, social media, and gambling (readiness/ confidence ruler (10 = "not at all ready/ difficult to change" to $100=$ "very 
ready/ difficult to change") assessing readiness and confidence in quitting or reducing each behavioral outcome; based on [38])

For a list of included secondary outcomes see Figure 2. Additionally, we assess socio-demographic data (age, gender, migration background, socio-economic status), progress of vocational education, educational sector and frequency of in-school education.

\section{Participant timeline $\{13\}$}

In Fig. 1, trial procedures from enrollment to the end of the trial are illustrated.

\section{Sample size $\{14\}$}

The sample size is calculated to detect a minimum relative difference of $20 \%$ between both groups at post-assessment. This effect was based on previous studies on the effectiveness of substance use prevention programs $[11,39]$ and takes a clustered-data structure and an intra-class correlation (ICC) of 0.032 as well as a drop-out rate of $30 \%$ on student level into account (based on [15]). The required sample size for $80 \%$ power to detect between-group differences at the 0.05 level is 4,500 students $(2,250$ students per condition) from 225 classes.

\section{Recruitment $\{15\}$}

The three study sites cooperate with local school supervisory authorities, which support the project and allow recruitment of a convenience sample of vocational schools. Class teachers and other school staff (such as school social workers) at those schools are informed about the study in teacher conferences. This information includes access to the project website which features explanation videos about the MZo-app and the implementation of the study. Teachers will be provided a login-code to test the MZo-app prior to implementation in their class. If teachers decide to participate in the study, two appointments for data collection and for the introduction of the intervention will be scheduled. Consent to participate in the study on student level has to be provided prior to baseline assessment.

\section{Assignment of interventions: allocation}

\section{Sequence generation $\{16 \mathrm{a}\}$}

Randomization will be performed at class level. The randomization sequence has been created [40] using the program „Randomization in Treatment Arms” (https://www.evidat.com/rita). Classes of vocational schools will be stratified by class characteristics and paired into dyads of similar classes. The first member of a dyad will have a $50 \%$ chance to be assigned to the intervention group, the remaining class will automatically be assigned to the other trial arm.

\section{Concealment mechanism $\{16 \mathrm{~b}\}$}


In the temporal order of study inclusion, allocation of classes will be made according to the randomization plan to either the MZo-intervention or the control group. Participants, teachers, and schools will have no influence on the allocation process and assignment of classes to either group but they will not be blind to randomization.

\section{Implementation $\{16 \mathrm{c}\}$}

Random allocation of classes within schools to either the MZo-intervention or the control group using the randomization plan takes place at each study site under the responsibility of the local study team. All students who give their written consent (electronically) to participate in the study, will be enrolled. Unique identifiers are generated using an ID-generator software and will provide access to the electronic assessment portal. The flow diagram of the study design is depicted in Fig. 1.

\section{Assignment of interventions: Blinding}

\section{Who will be blinded $\{17 a\}$}

All participants, teachers and schools are blinded to the randomization and allocation process. However, they will not be blind to the MZo-intervention, as participants of the control group will be informed that they will receive access to the MZo-app after post-assessment.

\section{Procedure for unblinding if needed $\{17 \mathrm{~b}\}$}

There is no need for unblinding.

\section{Data collection and management}

\section{Plans for assessment and collection of outcomes $\{18 \mathrm{a}\}$}

Data will be collected class-wise in schools. The baseline assessments will take place directly before the intervention, the post assessments at least 30 days after the end of the intervention. All measures are assessed via the online system "SoSci Survey", program version 3.2.12 (15.02.2021). All collected data is entered using participant's personal smartphones. If individual smartphones are unavailable or not functioning due to technical problems the study teams will provide tablets to ensure data collection. To guarantee a smooth online data assessment, the study teams will also provide a portable LTE-router for a stable internet connection in the classroom if necessary. As for the MZo-app and the online assessment portal, compatibility with current Android and iOS devices is given.

\section{Plans to promote participant retention and complete follow-up $\{18 \mathrm{~b}\}$}

According to their allocation, study participants will complete the online questionnaires in class. Unique identifiers are distributed by the study team and can be used to access the questionnaire for pre- and post-assessments. The same identifier can also be used to log-in to the MZo-app. To maintain high retention rates between pre- and post-assessments, participants are encouraged to take a smartphone 
picture of their unique identifier. To maximize data completeness, vocational students who are absent from school at the day(s) of the assessment will be contacted in a coordinated approach by their teachers.

\section{Data management $\{19\}$}

Online assessment data will automatically be transferred to a local server at the study site in Kiel (SH), minimizing errors of data entry. All participant data is handled in accordance with the General Data Protection Regulation (GDPR). All data will be maintained confidentially before, during, and after the trial and is stored securely at the study site in Kiel with access only by dedicated study team members.

\section{Confidentiality $\{27\}$}

All data is collected using 6-digit unique identifiers to access the online questionnaires. The first two digits of this code identify the specific school, the next two digits identify a particular class. The last two digits that identify an individual participant are randomly distributed. Single individuals cannot be identified.

\section{Plans for collection, laboratory evaluation and storage of biological specimens for genetic or molecular analysis in this trial/future use $\{33\}$}

No biological specimens will be collected.

\section{Statistical methods}

\section{Statistical methods for primary and secondary outcomes $\{20 \mathrm{a}\}$}

Analyses will account for clustering of individuals in school classes and will be reported following CONSORT standards. Trial variables will be analyzed by intervention arm, into which participants were randomly assigned. Continuous outcomes will be reported for each trial arm using means and standard deviations and binary outcomes will be reported for each trial arm using numbers and percentages. The main intervention effects will be tested by means of logistic or linear multi-level/ random effects regression models with the levels "classes" and "individuals", whereby group and time variables as well as the interaction term group $\mathrm{x}$ time are used. Primary between-group analyses will be adjusted for baseline scores of the outcome variable, relevant covariates (baseline prognostic factors that are theoretically associated with outcomes, including, but not limited to age, gender, and migration background as well as the personality traits sensation seeking and impulsiveness) and those variables used to stratify randomization.

\section{Interim analyses $\{21 \mathrm{~b}\}$}

No interim analysis will be performed.

Methods for additional analyses (e.g. subgroup analyses) $\{20 \mathrm{~b}\}$ 
For sensitivity analysis unadjusted and complete case (without imputation of missing data) analyses will additionally be carried out for primary and secondary outcomes. Potential moderators, such as individual differences in sociodemographic factors (age, sex, etc.) or vocational school-level factors (i.e., vocational sector etc.), and potential mediators (mechanisms of action) such as self-efficacy or abstinence/ reduction related control beliefs will be explored in interaction analyses.

\section{Methods in analysis to handle protocol non-adherence and any statistical methods to handle missing data $\{20 \mathrm{c}\}$}

Primary analyses will be based on the intention-to-treat population, thus including data from all participants who provide baseline data within a school class that was previously randomly assigned to one of the two trial conditions. Multiple imputation methods will be used to estimate missing data.

\section{Plans to give access to the full protocol, participant level-data and statistical code $\{31 \mathrm{c}\}$}

Anonymized study data and statistical codes will be made available on request given that data protection according to GDPR and ethics according to ethical approval is ensured.

\section{Oversight and monitoring}

\section{Composition of the coordinating centre and trial steering committee $\{5 \mathrm{~d}\}$}

The coordinating centre is the primary sponsor of this trial and responsible for study supervision. The coordinating investigators at the three study sites form a steering committee for the study. They meet regularly and are responsible to critically review the study design, the study protocol, the data management, and all study-related documents. The coordinating investigators provide oversight on the trial and support the study team members who conduct and provide day to day organisational support for the trial at each site. Furthermore, the coordinating investigators are responsible for the trial registration, revisions of the study protocol and application/amendments to the ethics committee, and scheduling of regular team meetings. All involved investigators and team members ensure compliance with the study protocol.

\section{Composition of the data monitoring committee, its role and reporting structure $\{21 \mathrm{a}\}$}

Not applicable, this trial is not monitored.

\section{Adverse event reporting and harms $\{22\}$}

Information on the occurrence of adverse events will be documented. Adverse events related to the assessment or use of the MZo-app during the 2-week intervention period will be recorded by the coordinating investigator.

Frequency and plans for auditing trial conduct $\{23\}$ 
Not applicable, there will be no external auditing during the trial.

\section{Plans for communicating important protocol amendments to relevant parties (e.g. trial participants, ethical committees) $\{25\}$}

All protocol deviations or modifications will be documented; all substantial protocol amendments will be communicated to the ethics committee of the Center for Psychosocial Medicine at the University Medical Center Hamburg-Eppendorf, the responsible school authorities at each study site (Center for Education Monitoring and Quality Development at schools in Hamburg, IfBQ; the Center for Prevention at the Institute for Quality Development at Schools in Schleswig-Holstein, IQ.SH; and the Bavarian State Ministry for Education and Cultural Affairs) and the German clinical trial register, DRKS (DRKS00023788).

\section{Dissemination plans $\{31$ a $\}$}

The study results will be presented at conferences and symposia and will be submitted for publication in relevant journals.

\section{Discussion}

The present study aims to transfer the voluntary abstinence approach that has been established in regular schools to the vocational school setting and extends it to digital media-related addictive behaviors. This will be the first time that a prevention program for vocational schools will be developed and rigorously evaluated in a randomized study in Germany. Moreover, the scientific evaluation of a fully app-based intervention of this approach extends the literature of prevention measures based on voluntary commitment which up to now has been mainly established in the form of the class competition "Be smart - don't start" [18].

If the trial results suggest that the approach is feasible and effective, this could provide a much needed evidence-based, relatively low-cost and scalable program to reduce substance use and internet-related problems at vocational schools in Germany. All MZo-materials (explanation video, app, MZo-website) is designed to require minimal effort and resources for schools/ teachers. The "core intervention material" is the MZo-app which can be considered credible for the target group since vocational students participated in the process of conceptual development to pilot-testing for usability and acceptability.

To facilitate recruitment of vocational schools, the cluster RCT-design is limited to a convenience sample of vocational schools willing to participate and a pre-post design. While this approach may result in selection bias and limit conclusions regarding long-term effectiveness, we consider this approach acceptable given that the study evaluates the effectiveness of a newly developed intervention. Although the recruited sample cannot be considered representative for vocational schools in Germany, generalizability of the results is facilitated by the inclusion of a broad range of vocational sectors (i.e., service industries, business and administrative professions as well as industrial-technical professions) within schools from three different states across Germany. Moreover, implications for practice will be 
gained. The study will contribute to the knowledge on how to effectively implement the MZo-intervention in (vocational) schools. Finally, the inclusion of a range of potentially relevant secondary outcomes, covariates, moderators and mediators in a reasonably large sample is expected to add to a differential understanding of the trial results.

\section{Trial status}

Ongoing trial. Recruitment of participants started in March 2021 and is expected to be completed in April 2022. Protocol version \#1; protocol version date 22 December 2021.

\section{Abbreviations}

ASKU: Short Scale for Measuring General Self-efficacy Beliefs

AUDIT - C: Alcohol Use Disorders Identification Test

BBGS: Brief Biosocial Gambling Screen

BFAS: Bergen Facebook Addiction Scale

BMG: Federal Ministry of Health Germany

CAMM: Child and Adolescent Mindfulness Measure

CG: Control condition (Group)

CONSORT: Consolidated Standards for Reporting Trials

DASS-21: Depression Anxiety Stress Scales 21

DRKS: German clinical trial register

GDPR: General Data Protection Regulation

ICC: Intra-class correlation

ICD-11: International Classification of Diseases

IfBQ: Center for Education Monitoring and Quality Development at schools in Hamburg

IG: Intervention Group

IGDS-SF: Internet Gaming Disorder Scale-Short-Form

IQ.SH: The Center for Prevention at the Institute for Quality Development at Schools in Schleswig-Holstein L1: General Life Satisfaction Short Scale 
MZo: „Meine Zeit ohne“ [My time without]

PMH: Positive Mental Health-scale

RCT: Randomized controlled trial

SDS: Severity of Dependence Scale

SPIRIT: Standard Protocol Items: Recommendations for Interventional Trials

SURPS: Substance Use Risk Profile Scale

WHO: World Health Organization

\section{Declarations}

\section{Acknowledgements}

We would like to acknowledge Simone Leuckfeld, Freia Biedenweg, Laura Borwieck and Kristin Grahlher for their contributions to the study. We also thank Regina Henkis, Gisela Mohr, Bernd Stüben, Armin Grzybek and all other persons involved in supporting the present study.

\section{Authors' contributions $\{31 \mathrm{~b}\}$}

$\mathrm{MM}, \mathrm{RH}, \mathrm{NA}, \mathrm{RT}, \mathrm{KL}$ and LK conceived the study, initiated study design and obtained funding. MM and $\mathrm{BP}$ are responsible for data management. MM, NA, KL, LK, JW, MR, BP developed the methodology for the intervention app and all study materials. NA and JW drafted the manuscript. All authors commented on the drafts of the manuscript and read and approved the final manuscript.

\section{Funding $\{4\}$}

The trial is funded by the Federal Ministry of Health Germany (BMG, grant number: ZMVI1-2519DSM216). Funding period is 01.08 .2019 to 31.12 .2022 . The funding source has no role in the design of this study and will not have any role during its execution, analyses, interpretation of the data, or decision to submit results.

\section{Availability of data and material $\{29\}$}

Study material (information sheets) is available to the public and can be found on the following website http://www.meine-zeit-ohne.de and by request. The MZo application is suitable for iOS (iOS 11.0 or newer) and Android (version 6 or newer) and is available in the app store for iOS devices and Play Store for Android devices. Anonymized study data and statistical codes to analyses may be made available on request following study closure. 
Approval for the study was obtained from the ethics committee of the Center for Psychosocial Medicine at the University Medical Center Hamburg-Eppendorf and the responsible school authorities at each study site (Center for Education Monitoring and Quality Development at schools in Hamburg, IfBQ; the Center for Prevention at the Institute for Quality Development at Schools in Schleswig-Holstein, IQ.SH; and the Bavarian State Ministry for Education and Cultural Affairs) prior to data collection. The study is conducted in accordance with CONSORT guidelines and complies with the principles laid down in the Declaration of Helsinki [41]. It is registered in the DRKS public database (trial registration number DRKS00023788). Written informed consent will be obtained from all participants prior to study enrollment. A model consent form can be provided on request. All substantial protocol deviations or modifications will be communicated to the Ethics Committee and DRKS register.

\section{Consent for publication $\{32\}$}

Model consent forms will be provided on request.

\section{Competing interests $\{28\}$}

The authors declare they have no conflict of interest.

\section{References}

1. Stockings $E$, Hall WD, Lynskey M, et al. Prevention, early intervention, harm reduction, and treatment of substance use in young people. The Lancet Psychiatry 2016; 3: 280-296.

2. Mokdad AH, Forouzanfar MH, Daoud F, et al. Global burden of diseases, injuries, and risk factors for young people's health during 1990-2013: a systematic analysis for the Global Burden of Disease Study 2013. The Lancet 2016; 387: 2383-2401.

3. Pabst A, Kraus L, Matos EG de, et al. Substanzkonsum und substanzbezogene Störungen in Deutschland im Jahr 2012. Sucht 2013; 59: 321-331.

4. Arnett JJ. Adolescent storm and stress, reconsidered. American psychologist 1999; 54: 317.

5. Jordan CJ, Andersen SL. Sensitive periods of substance abuse: Early risk for the transition to dependence. Developmental cognitive neuroscience 2017; 25: 29-44.

6. Wartberg L, Kriston L, Thomasius R. The Prevalence and Psychosocial Correlates of Internet Gaming Disorder. Dtsch Arztebl Int 2017; 114: 419-424.

7. Mann K, Fauth-Bühler M, Higuchi S, et al. Pathological gambling: a behavioral addiction. World Psychiatry 2016; 15: 297.

8. Brand M, Wegmann E, Stark R, et al. The Interaction of Person-Affect-Cognition-Execution (I-PACE) model for addictive behaviors: Update, generalization to addictive behaviors beyond internet-use disorders, and specification of the process character of addictive behaviors. Neuroscience \& Biobehavioral Reviews 2019; 104: 1-10. 
9. Dong $\mathrm{H}$, Yang F, Lu X, et al. Internet addiction and related psychological factors among children and adolescents in China during the coronavirus disease 2019 (COVID-19) epidemic. Frontiers in Psychiatry 2020; 11: 751.

10. Arnaud N, Thomasius R. Störungen durch Substanzgebrauch und abhängige Verhaltensweisen in der ICD-11. Zeitschrift für Kinder-und Jugendpsychiatrie und Psychotherapie.

11. Onrust SA, Otten R, Lammers J, et al. School-based programmes to reduce and prevent substance use in different age groups: What works for whom? Systematic review and meta-regression analysis. Clinical Psychology Review 2016; 44: 45-59.

12. Morgenstern M, Seidel A-K, Hanewinkel R. Suchtprävention an beruflichen Schulen. Bildung und Beruf 2019; 2: 50-57.

13. Bray JW, Galvin DM, Cluff LA. Young Adults in the Workplace: A Multisite Initiative of Substance Use Prevention Programs. RTI Press Book. RTI International.

14. Uhly A. A Indikatoren zur beruflichen Ausbildung A5 Ausbildung im dualen Ausbildungssystem Analysen auf Basis der Berufsbildungsstatistik. Datenreport, https://www.bibb.de/datenreport/de/2017/63477.php (2017, accessed 13 December 2021).

15. Montag J, Hanewinkel R, Morgenstern M. Verbreitung und Korrelate des Substanzkonsums unter 5 688 Auszubildenden an beruflichen Schulen. Das Gesundheitswesen 2015; 77: 411-417.

16. Kaess M, Parzer P, Brunner R, et al. Pathological Internet Use Is on the Rise Among European Adolescents. Journal of Adolescent Health 2016; 59: 236-239.

17. Wartberg L, Kriston L, Thomasius R. The Prevalence and Psychosocial Correlates of Internet Gaming Disorder. Dtsch Arztebl Int 2017; 114: 419-424.

18. Hanewinkel R, Wiborg G, Abdennbi K, et al. European smokefree class competition: a measure to decrease smoking in youth. Journal of epidemiology and community health 2007; 61: 750.

19. Isensee B, Hanewinkel R. Tabakprävention im Setting Schule am Beispiel von „Be smart-don't start “. Bundesgesundheitsblatt-Gesundheitsforschung-Gesundheitsschutz 2018; 61: 1446-1452.

20. Kalke J, Raschke P, Buth S. Initiierte Abstinenz. Prävention und Gesundheitsförderung 2006; 1: 219226.

21. Brailovskaia J, Ströse F, Schillack H, et al. Less Facebook use-More well-being and a healthier lifestyle? An experimental intervention study. Computers in Human Behavior 2020; 108: 106332.

22. Rumpf H-J. Entwicklung und Validierung eines ökonomischen Screening-Verfahrens zur Entdeckung von Alkoholabhängigkeit und -missbrauch in der medizinischen Basisversorgung.

23. Pontes HM, Griffiths MD. Measuring DSM-5 Internet gaming disorder: Development and validation of a short psychometric scale. Computers in human behavior 2015; 45: 137-143.

24. Montag C, Schivinski B, Sariyska R, et al. Psychopathological symptoms and gaming motives in disordered gaming-A psychometric comparison between the WHO and APA diagnostic frameworks. Journal of Clinical Medicine 2019; 8: 1691. 
25. Andreassen CS, Torsheim T, Brunborg GS, et al. Development of a Facebook addiction scale. Psychological reports 2012; 110: 501-517.

26. Brailovskaia J, Margraf J. Facebook Addiction Disorder (FAD) among German students-A longitudinal approach. PLOS ONE 2017; 12: e0189719.

27. Martin G, Copeland J, Gates P, et al. The Severity of Dependence Scale (SDS) in an adolescent population of cannabis users: Reliability, validity and diagnostic cut-off. Drug and Alcohol Dependence 2006; 83: 90-93.

28. Steiner S, Baumeister SE, Kraus L. Severity of Dependence Scale: Establishing a cut-off point for cannabis dependence in the German adult population. SUCHT 2008; 54: 57-63.

29. Gebauer L, LaBrie R, Shaffer HJ. Optimizing DSM-IV-TR classification accuracy: A brief biosocial screen for detecting current gambling disorders among gamblers in the general household population. The Canadian Journal of Psychiatry 2010; 55: 82-90.

30. Lovibond PF, Lovibond SH. The structure of negative emotional states: Comparison of the Depression Anxiety Stress Scales (DASS) with the Beck Depression and Anxiety Inventories. Behaviour research and therapy 1995; 33: 335-343.

31. Nilges P, Essau C. Die Depressions-Angst-Stress-Skalen: Der DASS - ein Screeningverfahren nicht nur für Schmerzpatienten. Schmerz 2015; 29: 649-657.

32. Woicik PA, Stewart SH, Pihl RO, et al. The substance use risk profile scale: a scale measuring traits linked to reinforcement-specific substance use profiles. Addictive behaviors 2009; 34: 1042-1055.

33. Greco LA, Baer RA, Smith GT. Assessing mindfulness in children and adolescents: development and validation of the Child and Adolescent Mindfulness Measure (CAMM). Psychological assessment 2011; 23: 606.

34. Lukat J, Margraf J, Lutz R, et al. Psychometric properties of the positive mental health scale (PMHscale). BMC psychology 2016; 4: 1-14.

35. Cheung F, Lucas RE. Assessing the validity of single-item life satisfaction measures: results from three large samples. Qual Life Res 2014; 23: 2809-2818.

36. Nießen D, Groskurth K, Rammstedt B, et al. Zusammenstellung sozialwissenschaftlicher Items und Skalen (ZIS). An English-language adaptation of the General Life Satisfaction Short Scale (L-1), https://zis.gesis.org/Doild/zis284 (2020, accessed 21 December 2021).

37. Beierlein $C$, Kovaleva A, Kemper $C$, et al. Zusammenstellung sozialwissenschaftlicher Items und Skalen (ZIS). Allgemeine Selbstwirksamkeit Kurzskala (ASKU), https://zis.gesis.org/Doild/zis35 (2014, accessed 21 December 2021).

38. Miller WR, Rollnick S. Motivational interviewing: helping people change. 3rd ed. New York, NY: Guilford Press, 2013.

39. Kalke J, Raschke P, Buth S. Initiierte Abstinenz. Prävention und Gesundheitsförderung 2006; 1: 219226. 
40. Pahlke F, König IR, Ziegler A. Randomization in treatment arms (RITA): Ein RandomisierungsProgramm für klinische Studien. Informatik, Biometrie und Epidemiologie in Medizin und Biologie, 2004, pp. 1-22.

41. World Medical Association Declaration of Helsinki: ethical principles for medical research involving human subjects. JAMA 2013; 310: 2191-2194.

\section{Figures}
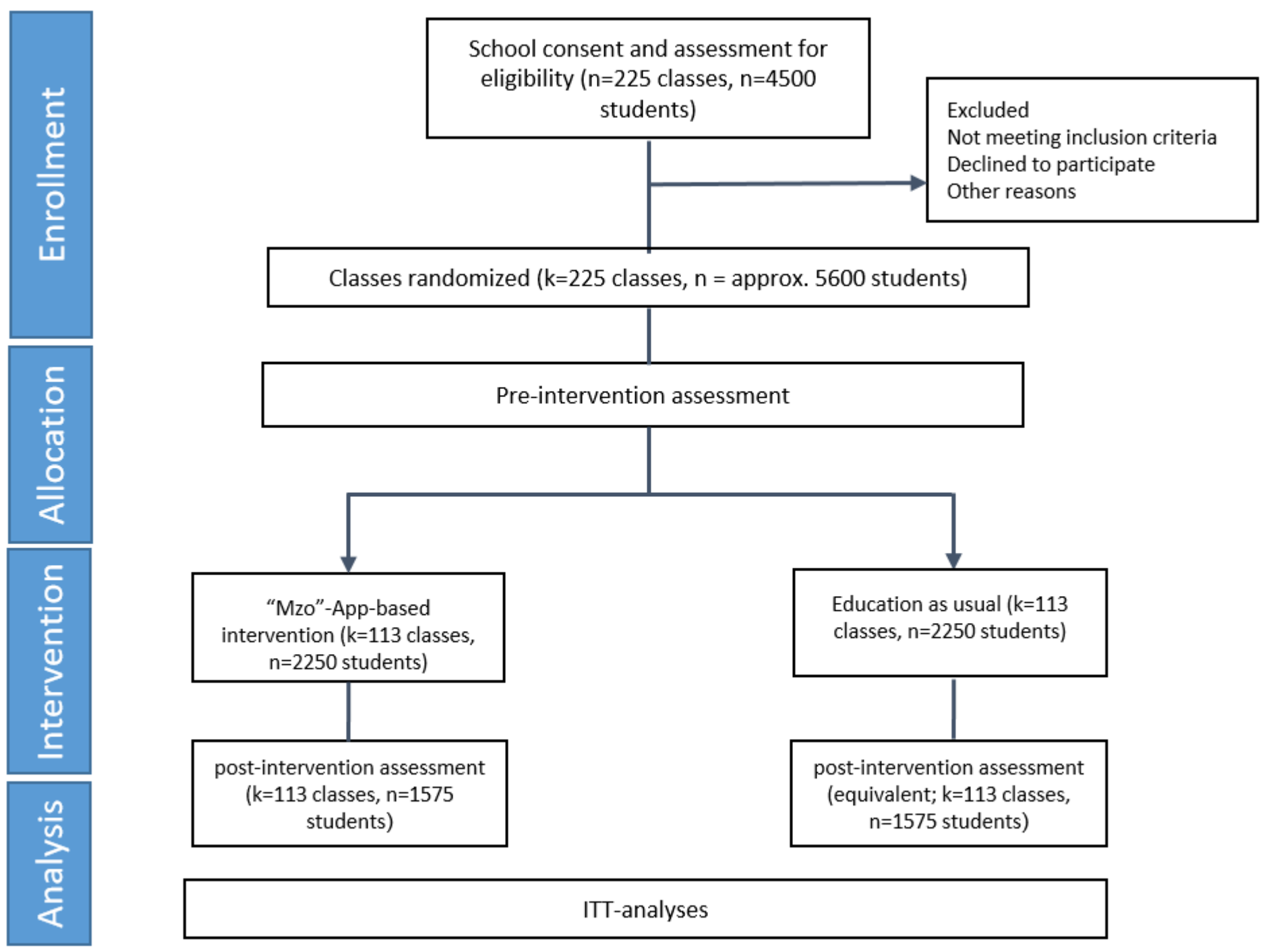

Figure 1

Consolidated Standards for Reporting Trials (CONSORT) diagram: study design and participant flow. 


\begin{tabular}{|c|c|c|c|c|c|}
\hline \multirow[b]{3}{*}{ TIMEPOINT } & \multicolumn{5}{|c|}{ STUDY PERIOD } \\
\hline & \multirow{2}{*}{$\begin{array}{c}\text { Enrolment } \\
t_{0}\end{array}$} & \multirow{2}{*}{$\begin{array}{c}\text { Allocation } \\
0 \\
\end{array}$} & \multicolumn{3}{|c|}{ Post-allocation } \\
\hline & & & Intervention & $t_{1}$ & Close-out \\
\hline \multicolumn{6}{|l|}{ Enrollment } \\
\hline Eligibility screening & $\mathrm{x}$ & & & & \\
\hline Informed consent & $\mathrm{x}$ & & & & \\
\hline Randomization & & $\mathrm{x}$ & & & \\
\hline \multicolumn{6}{|l|}{ INTERVENTIONS: } \\
\hline Mzo-Challenge & & & $\mathrm{x}$ & & \\
\hline $\begin{array}{l}\text { Education as usual (waitlist control } \\
\text { group) }\end{array}$ & & & & & $x$ \\
\hline \multicolumn{6}{|l|}{ ASSESSMENTS: } \\
\hline Sociodemographic information & $\mathrm{x}$ & & & & \\
\hline Vocational school characteristics & $\mathrm{x}$ & & & & \\
\hline $\begin{array}{l}\text { Alcohol use (past month frequency } \\
\text { and quantity of drinking and past } \\
\text { month frequency of binge drinking, } \\
\text { adapted from AUDIT-C) }\end{array}$ & $\mathrm{x}$ & & & $\mathrm{x}$ & \\
\hline $\begin{array}{l}\text { Nicotine use (past month frequency } \\
\text { and quantity of cigarette and/ or e- } \\
\text { cigarette use) }\end{array}$ & $\mathrm{x}$ & & & $\mathrm{x}$ & \\
\hline $\begin{array}{l}\text { (Problematic) Internet gaming } \\
\text { (IGDS-SF) }\end{array}$ & $\mathrm{x}$ & & & $\mathrm{x}$ & \\
\hline $\begin{array}{l}\text { (Problematic) Social media use } \\
\text { (adapted BFAS) }\end{array}$ & $\mathrm{x}$ & & & $\mathrm{x}$ & \\
\hline (Problematic) Cannabis use (SDS) & $\mathrm{x}$ & & & $\mathrm{x}$ & \\
\hline (Problematic) Gambling (BBGS) & $\mathrm{x}$ & & & $\mathrm{x}$ & \\
\hline $\begin{array}{l}\text { Psychological Stress (DASS-21, } \\
\text { subscale stress) }\end{array}$ & $\mathrm{x}$ & & & $\mathrm{x}$ & \\
\hline Physical activity & $\mathrm{x}$ & & & $\mathrm{x}$ & \\
\hline Positive mental health (PMH-9) & $\mathrm{x}$ & & & $\mathrm{x}$ & \\
\hline Life satisfaction (L1) & $\mathrm{x}$ & & & $\mathrm{x}$ & \\
\hline Self-efficacy (ASKU) & $\mathrm{x}$ & & & $\mathrm{x}$ & \\
\hline Impulsivity (SURPS, subscale) & $\mathrm{x}$ & & & $\mathrm{x}$ & \\
\hline $\begin{array}{l}\text { Sensation seeking (SURPS, } \\
\text { subscale) }\end{array}$ & $x$ & & & $\mathrm{x}$ & \\
\hline Mindfulness (CAMM) & $\mathrm{x}$ & & & $\mathrm{x}$ & \\
\hline Satisfaction with school & $\mathrm{x}$ & & & $\mathrm{x}$ & \\
\hline Readiness to change & $\mathrm{x}$ & & & $\mathrm{x}$ & \\
\hline
\end{tabular}

\section{Figure 2}

Standard Protocol Items: Recommendations for Interventional Trials (SPIRIT) diagram detailing trial activities and measures and their timing. 